\title{
An Alternative Technique for Determining Gravimetric Particle Mass Deposition on Filter Substrate: The Particle Extraction Method
}

\author{
Charity Garland, Samantha Delapena, David Pennise \\ Berkeley Air Monitoring Group, Berkeley, CA, USA \\ Email: charityrose.g@gmail.com
}

How to cite this paper: Garland, C., Delapena, S. and Pennise, D. (2018) An Alternative Technique for Determining Gravimetric Particle Mass Deposition on Filter Substrate: The Particle Extraction Method. Open Journal of Air Pollution, 7, 309-321. https://doi.org/10.4236/ojap.2018.74016

Received: October 30, 2018

Accepted: December 22, 2018

Published: December 25, 2018

Copyright $\odot 2018$ by authors and Scientific Research Publishing Inc. This work is licensed under the Creative Commons Attribution International License (CC BY 4.0).

http://creativecommons.org/licenses/by/4.0/ (c) (i) Open Access

\begin{abstract}
Airborne particulate matter (PM) filter sample processing is susceptible to error and can present issues associated with organizing samples, tracking data, and maintaining weighing conditions. While filter weighing facilities should implement robust quality assurance and control checks to ensure that data collection is accurate and filter storage is secure, mistakes and accidents can still occur that compromise valuable data. This paper presents a novel approach to PM filter sample processing that allows for data validation or data recovery while ensuring data integrity. The technique approximates the original, unused pre-sampling weight of polytetrafluoroethylene (PTFE) filters after PM collection to determine PM mass-deposition (MD). The method describes the extraction of PM loaded on PTFE filters via sonication in relatively non-toxic solvents, methanol and distilled water. The extraction method is compared to the standard gravimetric PM MD determination method for a set of 265 PTFE filters with mean post-sampling filter mass of $116 \pm 3.6$ $\mathrm{mg}$, mean estimated PM MD using the standard method of $367 \pm 589 \mu \mathrm{g}$, and mean estimated PM MD using the extraction method of $371 \pm 589 \mu \mathrm{g}$. A Deming regression comparison of the two methods yields a slope of 0.9983 and a Pearson's $r$ of 0.999. A Bland-Altman assessment of the percent and absolute differences between the two methods shows the limits of agreement between $-32.5 \%$ and $25.5 \%$ and -61.9 and $50.1 \mu \mathrm{g}$, respectively. The $99 \%$ confidence interval of the mean difference in mass deposition between the two methods is $-5.8 \pm 4.5 \mu \mathrm{g}$. These data demonstrate that estimating pre-sampling PTFE filter mass by extracting PM from sampled filters is a viable technique for gravimetric filter analysis. This method is of use in recovering pre-sampling filter weights that have been lost, incorrectly measured, or otherwise compromised.
\end{abstract}




\section{Keywords}

PTFE Filters, Gravimetric Analysis, Particulate Matter, Particle Extraction, Gravimetry

\section{Introduction}

Gravimetric filter analysis, employing a pump and filter system for sample collection, is the gold standard method for measuring the mass concentration of particulate matter (PM) in air (Balakrishnan et al., 2015; Health and Safety Executive (HSE), 1997; Rosa et al., 2014) [1] [2] [3]. The basis for this analysis is rooted in high precision ( $0.1-1 \mu \mathrm{g}$ resolution) mass measurements of filter media taken prior to and after sample collection. High-precision scales used for weighing filters must be operated in a highly controlled environment that is clean, demonstrates low static and vibration, and is temperature and humidity stable, to ensure consistency in scale performance and accuracy of the measurement. Moreover, the conditions must be well-matched between pre- and post-sampling filter mass measurements, which may be separated by several months and up to years, making these requirements potentially difficult to satisfy. Additionally, filter data may be lost or compromised by incorrect mass measurements resulting from various issues including unstable scales, improper scale operation or calibration, or transcription errors. Large sample sets can be comprised of hundreds to thousands of filters and some erroneous mass measurements are likely to occur. While post-sampling filter mass measurements can be repeated if filters are stored properly, there is currently no method for recovering lost or compromised pre-sampling filter mass measurements.

This study examines the efficacy of a particle extraction method used to approximate the original, unused pre-sampling mass of commonly used polytetrafluoroethylene (PTFE) filters used for collection of PM smaller than $2.5 \mu \mathrm{m}$ in aerodynamic diameter $\left(\mathrm{PM}_{2.5}\right)$, from primarily wood smoke sources.

\section{Materials and Methods}

During standard gravimetric PM sample collection, filter media are precisely pre-weighed in a temperature and humidity-controlled room prior to deployment and sampling. A known volume of sample air is passed through the filter medium by a calibrated air sampling pump. The PM contained in the air is deposited on the pre-weighed filter during sampling. The filter is post-weighed after sampling using the same balance, ideally under the same conditions and with the same operator as the pre-sampling filter mass measurement. The difference between the filter pre-sampling weight and post-sampling weight yields the mass deposition (MD), or mass of PM that was deposited on the filter that was previously contained in the sampled air. The volume of the sampled air is logged by some samplers, otherwise it is calculated using the average of the pre-sampling 
and post-sampling flow rate of the pump (liters, L, per minute), multiplied by the duration of sampling (minutes). Dividing the PM mass $(\mu \mathrm{g})$ by the known volume of air (converted from $\mathrm{L}$ to $\mathrm{m}^{3}$ ) passed through the filter results in an average mass concentration of PM during the sampling period in terms of mass of PM per unit volume $\left(\mu \mathrm{g} / \mathrm{m}^{3}\right)$. The pump and filter method of sample collection and analysis is common and more detailed protocols exist (RTI International, 2008; USEPA, 2017) [4] [5].

Changes in filter mass unrelated to the PM mass collected during sampling, such as contamination or mass loss from filter handling and/or transport, are commonly accounted and corrected for by using field blank filters. These field blank filters generally number between $10 \%$ and $20 \%$ of the total number of sampled filters and are treated the same way as the sample filters, apart from sampling air, including all aspects of storage, transportation, and sample handling.

Particle extraction tests were performed on "traditional" pump and PTFE filter samples of $\mathrm{PM}_{2.5}$ household air pollution (HAP) and personal exposure (PE) from primarily wood fuel combustion from cook stoves in rural Lao People's Democratic Republic (Lao PDR) (L. Drew Hill et al., 2015) [6]. A total of 265 filters were analyzed by both the standard gravimetric method and the PM extraction method proposed herein.

\subsection{Materials}

\subsubsection{Filter Samples}

Filter samples were collected on $37 \mathrm{~mm}$ diameter, $2 \mu \mathrm{m}$ pore size PTFE filters with polymethylpentene (PMP) support rings (Pall Corporation). $\mathrm{PM}_{2.5}$ mass deposited on the filter was determined gravimetrically by weighing the filters on an electronic microbalance with $0.1 \mu \mathrm{g}$ resolution (Mettler Toledo XP2U, USA) (RTI International, 2008) [4]. All filters had known pre-sampling weights, duplicate post-sampling weights to ensure accuracy, and post-extraction weights. Filter samples were:

1) 265 sample filters with primarily wood smoke $\mathrm{PM}_{2.5}$ deposition from HAP and PE field samples. Of this group, six samples were excluded due to apparent incorrect pre-sampling weights, evidenced by negative mass depositions. An additional 28 filters were excluded that were below the extraction method's limit of detection of $60 \mu \mathrm{g}$, which is described in Section 2.3.3,

2) 21 field blanks (handled similarly to the sample filters but not used for sampling),

3) Eight extraction blanks (filters taken directly from the package and not otherwise handled or sampled), and

4) Four lab-simulated filter samples loaded with wood smoke PM deposited using an air pollution chamber.

\subsubsection{Extraction Materials}

Extraction was performed using two $600 \mathrm{~mL}$ sonicators (SRA TruPower UC-06D 
Digital Ultrasonic Cleaner). Filters were sonicated in $50 \mathrm{~mL}$ glass beakers, covered with $5 \mathrm{~cm}$ watch glasses. Methanol (ACS reagent, $\geq 99.8 \%$ ) and distilled water were used as extraction solvents.

\subsection{Particle Extraction}

While there is limited literature on the extraction of PM from filter media, one of the few relevant experiments analyzed the extraction efficiency of wood smoke particulates from PTFE coated glass-fiber filter media using various solvents. This study demonstrated that the highest mass recovery of PM resulted from the use of methanol (MeOH) as the solvent (Williams et al., 1988) [7]. Thus, our initial trials of PM extraction from PTFE filter substrate were completed using $\mathrm{MeOH}$.

Early tests of extraction efficiency and pre-sampling filter weight estimates were completed using lab-simulated filter loadings of three to six milligrams of PM on PTFE filters using an air pollution chamber containing smoke from wood combustion. The MD was determined using the post-sampling mass and both the original pre-sampling and post-extraction filter masses. The average MD derived from the two methods was found to be within $0.4 \%$ of one another $(\mathrm{N}=4)$. Extraction times were tested to optimize time and extraction effectiveness by comparing post-extraction filter mass and pre-sampling filter mass agreement. Post-extraction filter-mass reached a floor after three 150-second sonication baths, two completed in pure $\mathrm{MeOH}$ followed by one in pure distilled water. The first two baths in $30 \mathrm{~mL}$ of fresh $\mathrm{MeOH}$ removed all visible PM deposited on the filter. The third sonication bath in fresh distilled water was intended to remove any water-soluble PM material that remained on the filter. Following the final water bath, filters were briefly immersed in fresh $\mathrm{MeOH}$, to expedite drying, and placed in partially covered petri dishes. Filter conditioning duration was tested by mass measurements taken every 24 hours over four days. No changes in mass were observed after 48 hours, therefore conditioning in a humidity and temperature-controlled room lasted at least 48 hours. Once conditioned, the post-extraction filter mass was determined gravimetrically. All filters were treated using this particle extraction protocol.

The detailed protocol is as follows:

1) Equilibrate filters to room temperature from the refrigerator or freezer for approximately 45 minutes to reduce the risk of tearing;

2) Fill sonicator half full of water;

3) Place $50 \mathrm{~mL}$ beakers in sonicator;

4) Add $30 \mathrm{~mL}$ of $\mathrm{MeOH}$ to each beaker;

5) Place filter PM side down in beaker and sonicate for 75 seconds;

6) Flip the filter over with forceps and sonicate for another 75 seconds;

7) Remove filter and replace used $\mathrm{MeOH}$ with $30 \mathrm{~mL}$ of fresh $\mathrm{MeOH}$;

8) Sonicate the filter in both orientations for 75 seconds each;

9) Remove filter and replace dirty $\mathrm{MeOH}$ with $30 \mathrm{~mL}$ of fresh distilled water;

10) Sonicate the filter in both orientations for 75 seconds each; 
11) Remove filter and dip in fresh $\mathrm{MeOH}$ bath;

12) Place filter in slightly ajar petri dish and let dry and condition in a clean, temperature and humidity-controlled room for at least 48 hours;

13) Weigh filter according to typical weighing protocol.

\subsection{Data Handling}

\subsubsection{Blank Filter Processing}

While developing this method, unused filters underwent the extraction protocol to determine whether there was filter mass loss solely due to the extraction method. Eight extraction blanks were conditioned for 48 hours in a temperature and humidity-controlled room and then weighed prior to extraction. Extraction of new filters resulted in a loss, on average, of $21 \pm 13 \mu \mathrm{g}$. Since PTFE is not soluble in methanol it is possible that the new filters contain residue that was removed during extraction. Research Triangle Institute (RTI) has shown that off-gassing of filters can last longer than 48 hours after opening, which may result in changes in filter mass (RTI International, 2008) [4]. This finding emphasizes the importance of testing mass stability of new filter batches.

The blank filter processing test was used for determining the extraction method limit of detection (LOD) and was not used in calculating the post-extraction filter mass. Any filter mass loss incurred by the filters unrelated to PM deposition is accounted for by a post-extraction field blank filter adjustment, explained in the following section.

\subsubsection{Mass Deposition}

The average un-extracted, original field blank mass change $\left(F_{o}\right)$ was used to correct the original $\mathrm{MD}\left(M_{o}\right)$, as is standard to account for non-sampling-related filter mass changes during PM measurement campaigns (RTI International, 2008; USEPA, 2017) [4] [5]. Analogously, the average post-extraction field blank mass change $\left(F_{e X}\right)$ was used to apply a batch-specific correction to the extracted $\operatorname{MD}\left(M_{e x}\right)$ to obtain a realistic proxy for a filter's original MD $\left(M_{o}\right)$. Applying a batch-specific correction allowed for field blanks to act as a more specific proxy for filter mass changes caused by processes that vary between filter batches; such as shipment, field transport, and storage. The average field blank mass change from a given batch was subtracted from each sampled filter's MD in the batch to account for previously described changes in mass unrelated to sample collection, as well as differences between the pre-sampling $\left(X_{p r e}\right)$ and post-extraction $\left(X_{e x}\right)$ weights caused by the extraction process.

MD for the full filter data set was determined using both the standard gravimetric $\left(M_{o}\right)$ and the extraction $\left(M_{e x}\right)$ method employing the following calculations:

$$
\begin{aligned}
& \mathrm{M}_{o}=X_{p o s t}-X_{p r e}-F_{o} \\
& \mathrm{M}_{e x}=X_{p o s t}-X_{e x}-F_{e x}
\end{aligned}
$$




$$
\begin{aligned}
& F_{o}=\frac{\sum_{n=1\left(f_{b-p o s t}\right) n}^{N}{ }^{-}\left(f_{b-p r e}\right) n}{N} \\
& F_{e x}=\frac{\sum_{\left.n=1\left(f_{b-p o s t}\right)\right)^{n}}^{N}\left(f_{b-e x}\right) n}{N}
\end{aligned}
$$

where:

$M_{o}=$ original MD using original, un-extracted field blank adjustment.

$M_{e x}=$ extracted $\mathrm{MD}$ using post-extraction filter weight and post-extraction field blank adjustment.

$F_{o}=$ average field blank original mass change.

$F_{e x}=$ average field blank post-extraction mass change.

$X_{\text {post }}=$ sample filter post-sampling mass.

$X_{\text {pre }}=$ sample filter pre-sampling mass.

$X_{e x}=$ sample filter post-extraction mass (filter pre-sampling mass proxy).

$f_{b-\text { post }}=$ field blank post-sampling mass.

$f_{b-p r e}=$ field blank pre-sampling mass.

$f_{b-e x}=$ field blank post-extraction mass.

To help clarify the operational order of both the standard gravimetric and extraction methods, Figure 1 shows a schematic of filter handling and data analysis steps for sample and field blank filters for both processes.

\subsubsection{Limit of Detection}

Extraction blanks were analyzed to determine the limit of detection (LoD) for this method using the commonly accepted calculation for LOD shown in equation $5\left(y_{L O D}\right)$. The average mass change $\left(B_{e x}\right)$ of $21 \mu \mathrm{g}$ for all extraction blanks $(\mathrm{N}$ $=8)$ and the standard deviation of the extraction blank mass change $\left(\sigma_{B e x}\right)$ of 13 $\mu \mathrm{g}$ yielded an LoD of $60 \mu \mathrm{g}$. All samples with $M_{e x}$ below $60 \mu \mathrm{g}$ were excluded from the following analysis $(\mathrm{N}=28)$ for a total sample size of 231 .

$$
y_{L O D}=B_{e x}+3 * \sigma_{B e x}
$$

\section{Results and Discussion}

The average post-extraction field blank mass change $\left(F_{e x}\right)$ ranged from 15 to 21 $\mu \mathrm{g}$ depending on the sample batch, as compared to the original average field blank mass change $\left(F_{o}\right)$ of -2 to $-5 \mu \mathrm{g}$, meaning the post-extraction field blank mass was smaller than the original pre-weights by between 17 and $26 \mu \mathrm{g}$. This change in mass is consistent with the results of the blank filter extraction experiment, which showed a reduction in blank filter mass of about $21 \mu \mathrm{g}$ post-extraction.

Figure 2 depicts a Deming regression model relating the field blank adjusted original mass deposition $\left(M_{o}\right)$ and the post-extraction, field blank adjusted mass deposition $\left(M_{e x}\right)$ for the full sample set (A) and for filters with MD less than or equal to $1000 \mu \mathrm{g}$ (B). The Deming regression was used instead of a simple linear 
Standard Gravimetric

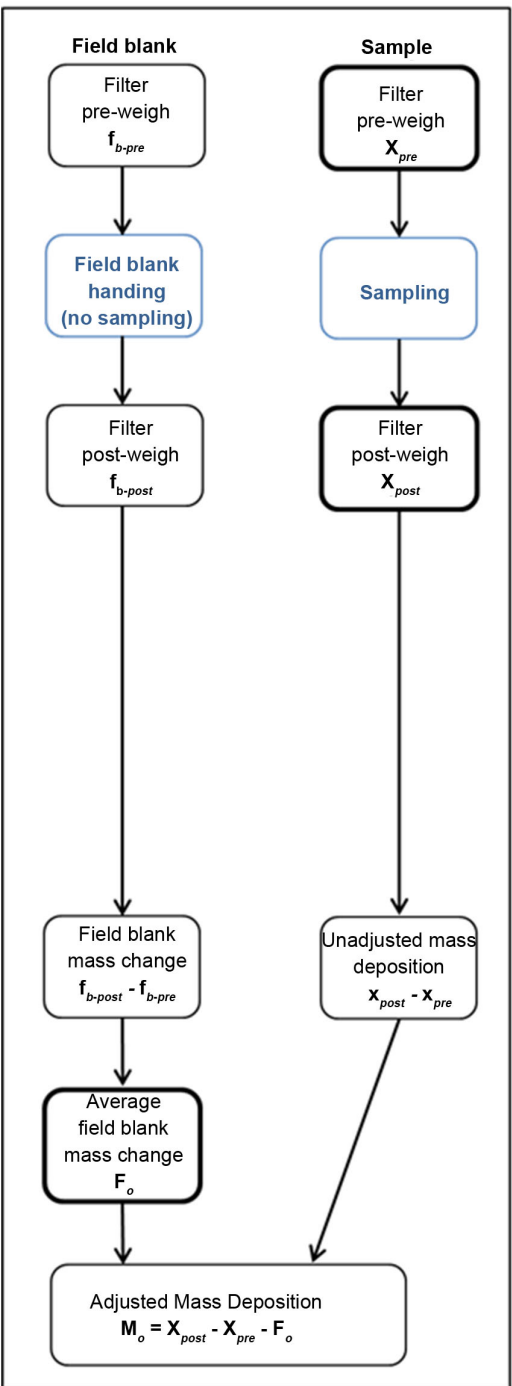

Extraction Method

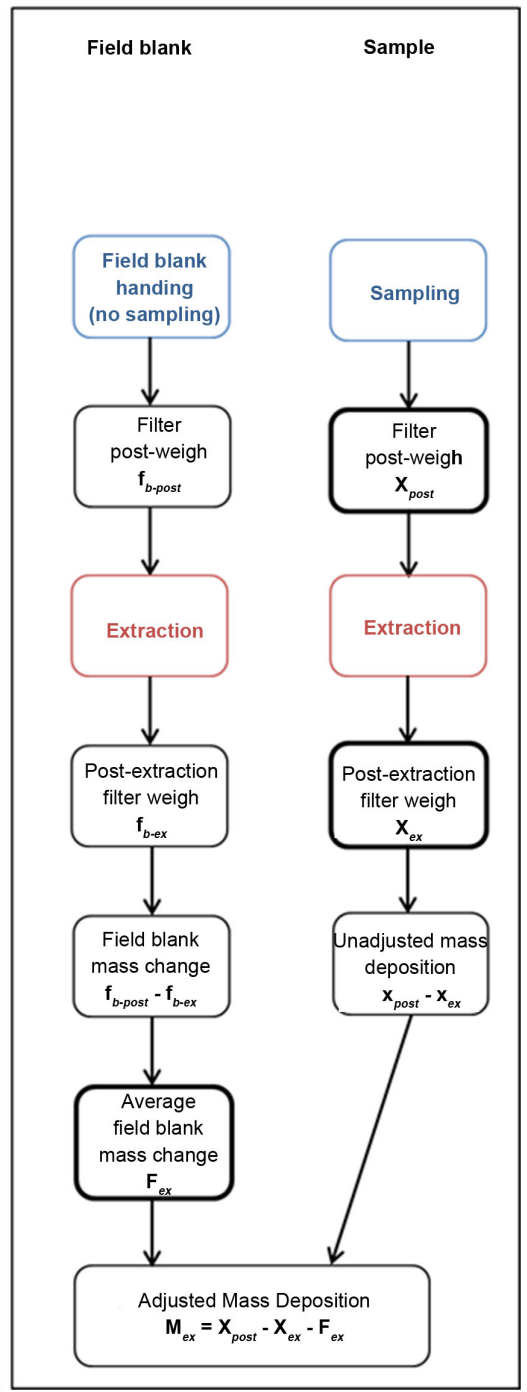

Figure 1. Filter handling and data analysis for the standard gravimetric and extraction method filter processing.

regression since it accounts for errors in both methods of a method comparison (Deming, W.E., 1943) [8]. The agreement between the two methods for the full filter set is excellent, with a slope of 0.998 and a Pearson's correlation coefficient (r) of 0.999. Examining only lower mass deposition filters with MD less than or equal to $1000 \mu \mathrm{g}(\mathrm{N}=216)$ shows the slope (1.0119) and the correlation coefficient $(r=0.991)$ to be similar to the values for the complete sample set, indicating that high MD outliers are not driving the relationship between the methods. The Deming regression analysis was done using $\mathrm{R}$ version 3.4.2. The package "mcr" was used for the analysis and visualization (Manuilova, E., et al., 2014) [9].

Figure 3 shows the distribution of the individual differences in mass deposition derived from the original pre-sampling weight $\left(M_{o}\right)$ and the post-extraction weight $\left(M_{e x}\right)$, both with the field blank adjustment applied. The $99 \%$ confidence 


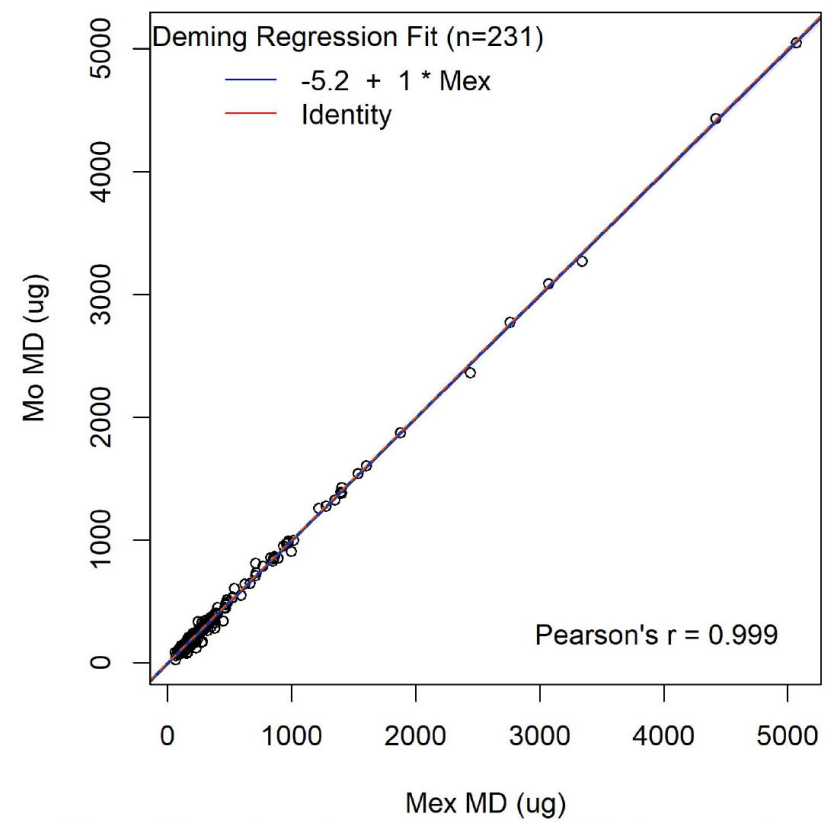

(a)

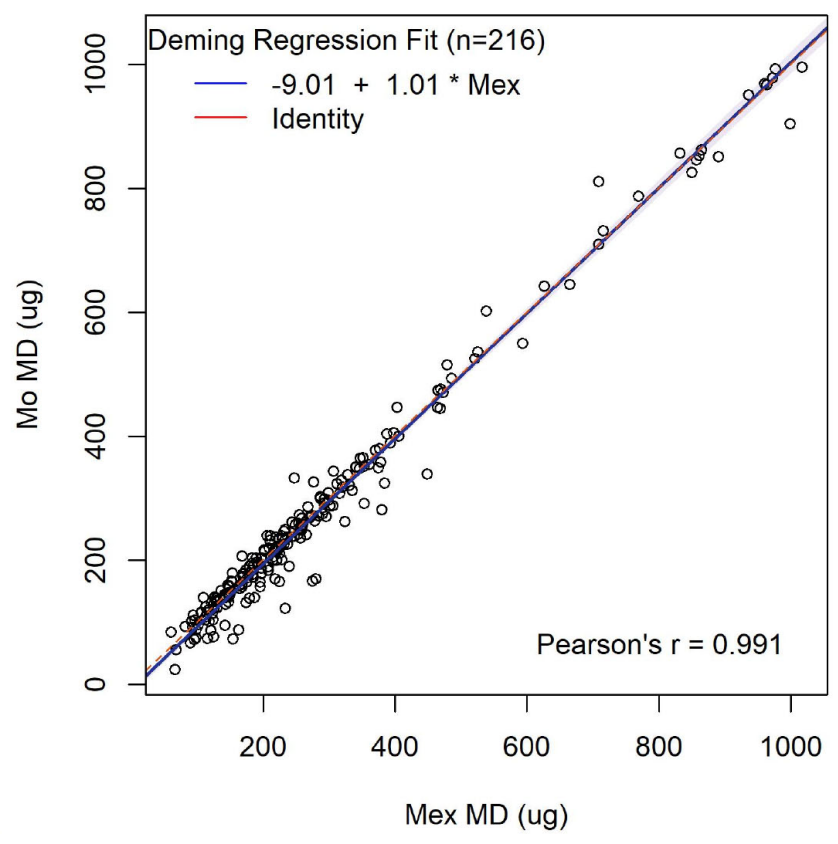

(b)

Figure 2. A Deming regression model relating original mass deposition from the standard gravimetric method $\left(M_{o}\right)$ versus mass deposition determined from the extraction method $\left(M_{e x}\right)$ for all filters (a) and for filters with an $M_{o}$ of $1000 \mu \mathrm{g}$ or less (b). The $95 \%$ confidence bounds are calculated with the bootstrap (quantile) method. The "Identity" line indicates equivalent methods.

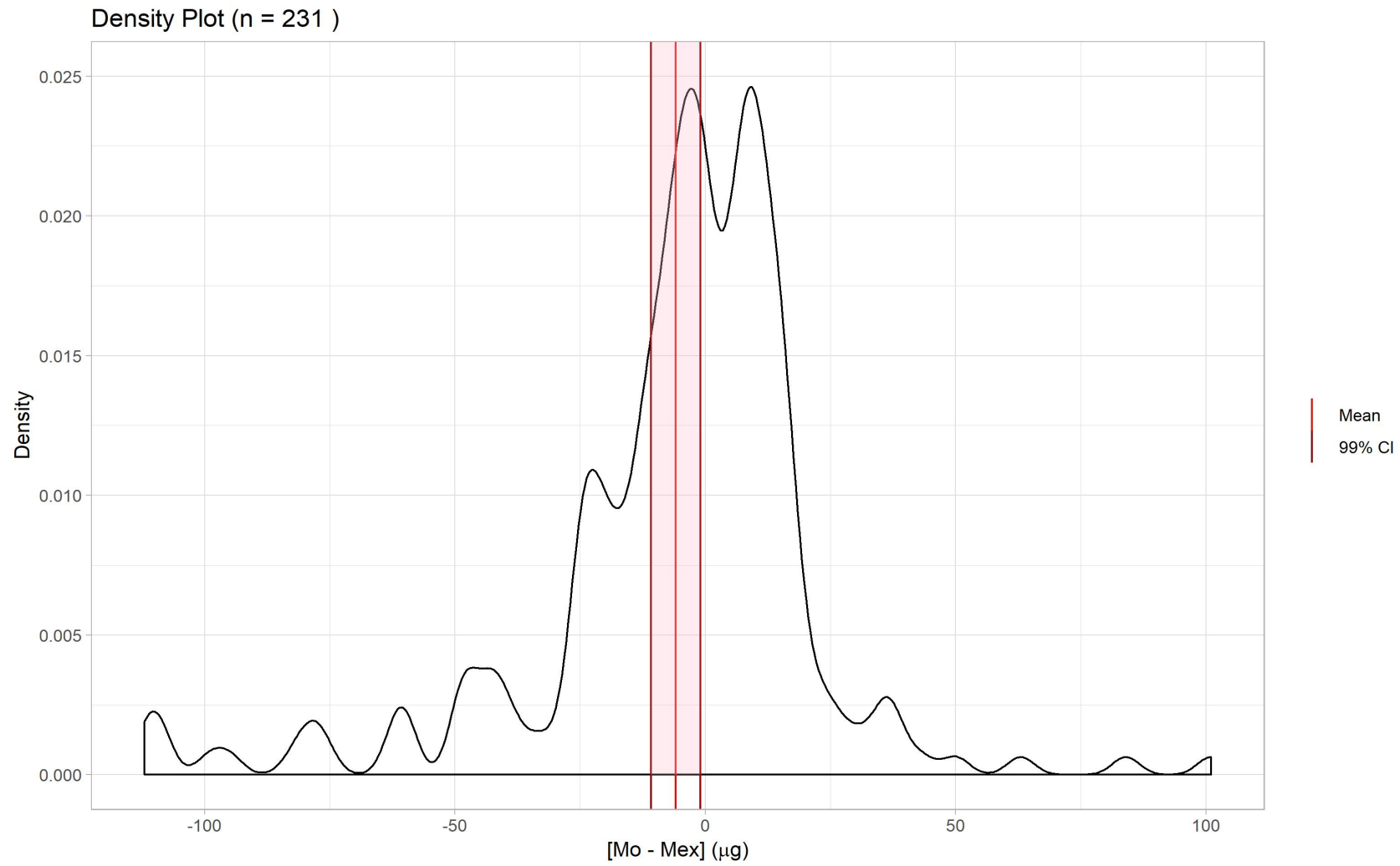

Figure 3. The mean mass deposition difference determined from field-blank adjusted original pre-sampling weight $\left[M_{o}\right]$ and field-blank adjusted post-extraction weights $\left[M_{e x}\right]$ is $-5.8 \pm 4.5$. The distribution of the differences in mass deposition using the two methods is shown with the $99 \%$ confidence interval and mean indicated by the vertical lines. 
interval (CI) for the set of measurement differences is $-5.8 \pm 4.5 \mu \mathrm{g}$, indicating there is a $99 \%$ chance that the average difference in MD from these two methods, if implemented as described herein, will be between $1.3 \mu \mathrm{g}$ and $10.3 \mu \mathrm{g}$ for the sample population. The upper and lower CIs for the set of MD differences account for between approximately $0.35 \%$ and $2.8 \%$, respectively, of the mean estimated PM MD for both the standard method (367 $\pm 589 \mu \mathrm{g})$ and the extraction method $(371 \pm 589 \mu \mathrm{g})$.

The Bland-Altman analysis is useful for comparing two different measurement methods that both have inherent error (Altman, D.G. and Bland, J.M., 1983) [10]. Figure 4(a) shows the absolute error between the two methods versus the average mass deposition using the two different methods. The mean absolute error is $-5.9 \mu \mathrm{g}$ and the limits of agreement (LoA) for the two methods are $\pm 56 \mu \mathrm{g}$, indicating $95 \%$ of the individual differences between $M_{o}$ and $M_{e x}$ lie between 50.1 and $-61.9 \mu \mathrm{g}$. The percent error in MD using the two methods versus the average of the two measurement methods is shown in Figure 4(b) and demonstrates that the limits of agreement (LoA) for the two methods are within $\pm 29 \%$ of the mean of $-3.5 \%$. As expected, the percent error gets larger as the measurement approaches the LoD. The percent error Bland-Altman plot indicates that few samples were outside the bounds of the LoA $(\mathrm{N}=12)$ and that they are all relatively low MD filters, approximately $280 \mu \mathrm{g}$ or less.

The mean values of the absolute $(-5.9 \mu \mathrm{g})$ and percent error $(-3.5 \%)$ indicate a slight bias between the two methods, with a minor overestimate by the extraction method. Correcting the bias by adding the mean difference of $-5.9 \mu \mathrm{g}$ to individual $M_{e x}$ values improved the estimate, yielding a mean absolute error of close to $0 \mu \mathrm{g}$. However, this adjustment is likely to vary from project to project and would be difficult to derive on a case by case basis. For this reason, we do not recommend making the mean absolute error adjustment when employing the extraction method unless a significant subset of representative samples throughout the study have both $M_{o}$ and $M_{e x}$ measurements to derive a representative value for approximate absolute error. In the case of this study, using the absolute error confidence interval of $9.7 \mu \mathrm{g}$ and a population size of 231 , a subset of $43 \%$ of the samples would allow a representative assessment of absolute error of this sample set. The Bland-Altman figures were created in $\mathrm{R}$ version 3.4.2. The package ggplot 2 was used to generate the figures.

To understand the magnitude of the effect of the extraction method on the metric of interest, an estimate of PM concentration was done using a typical gravimetric sample scenario for the standard and extraction MD determination methods, respectively. For the mass deposition sample set analyzed herein, a sample duration of 1440 minutes and a flow rate of 1.5 liters per minute was assumed, which is common for exposure and indoor air pollution studies. The difference in average approximate concentration using the two methods was found to be $2 \mu \mathrm{g} / \mathrm{m}^{3}$. The standard method yields an approximate concentration of $170 \pm$ $272 \mu \mathrm{g} / \mathrm{m}^{3}$ and the extraction method yields a concentration of $172 \pm 273 \mu \mathrm{g} / \mathrm{m}^{3}$. 


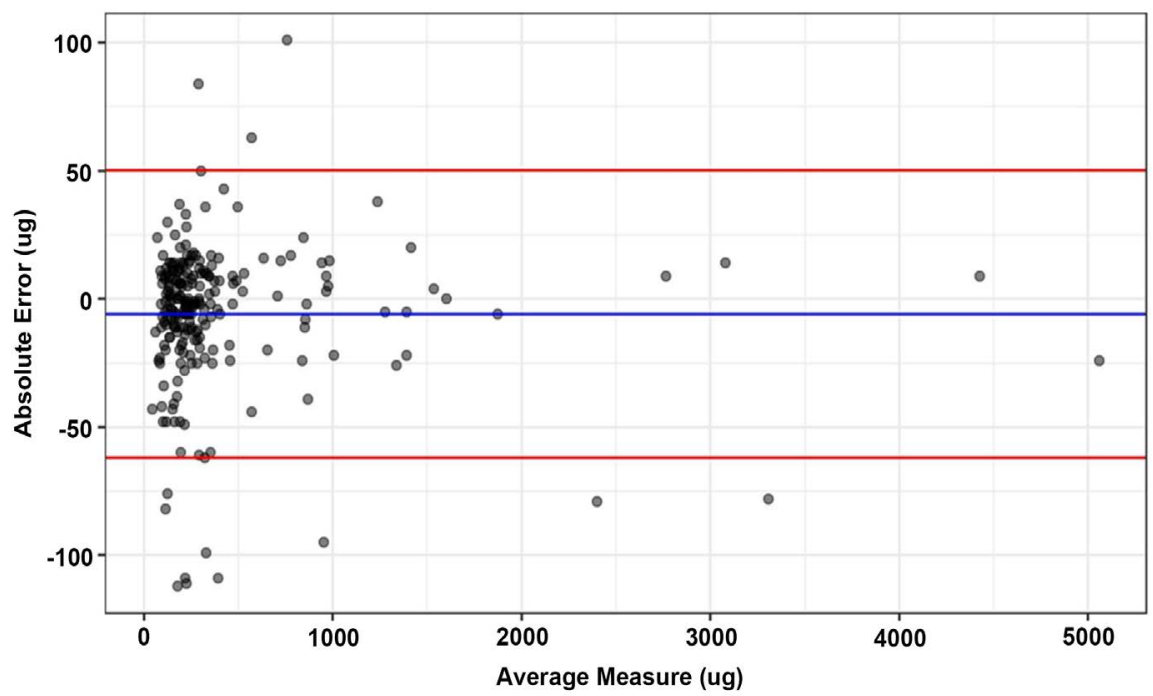

(a)

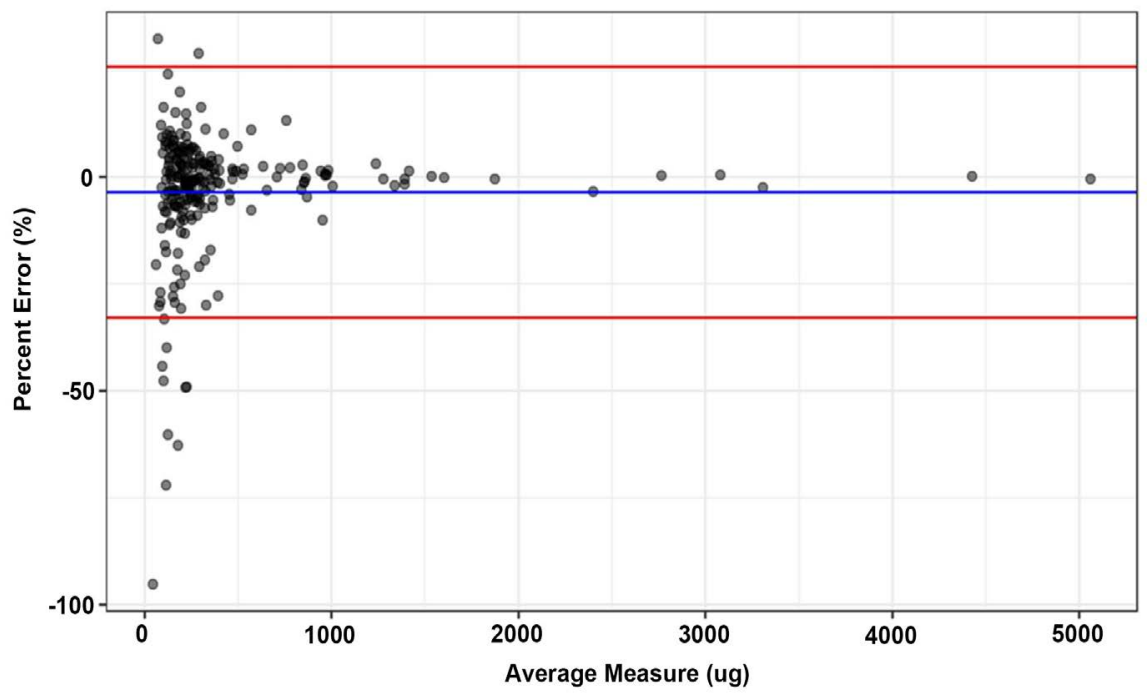

(b)

Figure 4. Bland-Altman relationships shows the absolute $\left(M_{o}-M_{e x}\right)$ (a) and percent (b) error between the two MD determination methods $\left(M_{o}\right.$ versus $\left.M_{e x}\right)$ for all values where $M_{e x}>$ LOD $(\mathrm{N}=231)$. The percent error is calculated using the average of the two measurements. The mean value is defined by the blue line and the $95 \%$ limits of agreement are shown in red [mean $\pm 1.96^{*}$ standard deviation] (A: $-5.9 \pm 56 \mu \mathrm{g}, \mathrm{B}:-3.5 \% \pm 29 \%$ ).

The data presented herein provide evidence that estimating pre-sampling filter mass from sampled PTFE filters by extracting wood smoke-derived PM during a sonicated $\mathrm{MeOH}$ bath is a viable method of gravimetric filter analysis, expressly when PM deposition is expected to be above $60 \mu \mathrm{g}$. This method efficaciously yields the MD of $\mathrm{PM}$, with the benefit of reducing the pre- and post-weighing interval, thereby minimizing variability in weighing conditions. The method is most valuable for recovering pre-sampling filter weights that are compromised, lost, unavailable, or thought to be incorrect, such as when MD calculations yield negative results. 


\section{Method Implementation Considerations}

While this process is robust, some considerations and limitations exist:

- Like standard gravimetric measurements, the analysis relies on field blanks to account for mass changes due to handling in the field and extraction. This method requires a minimum of ten field blanks, and ideally $10 \%-20 \%$ of total filter sample size. These field blanks also undergo the extraction process.

- The field blank adjustment will vary depending on the field campaign, filter size, and duration of filters in the field. Field blank analysis, such as described herein, should be done for any filter project attempting extraction.

- This process has only been tested on PTFE filters with PMP support rings. PTFE coated glass fiber filters and other types of filter media may not be viable for this type of filter processing. Care should be taken to undertake adequate testing prior to using the method on other types of filter media.

- Filters that are not equilibrated to room temperature before extraction are susceptible to ripping during the extraction process. Tearing did not result in statistically significant differences between $M_{o}$ and $M_{e x}(\mathrm{p}=0.17)$, and so torn filters were included in the analysis. However, ensuring that the filters are at room temperature and not placed directly from the refrigerator into the sonicator will reduce the likelihood of tearing during extraction.

- Extracted filters proved to be relatively more electrically charged than unextracted filters. It is recommended to use all possible anti-static measures when weighing extracted filters to reduce measurement time and error.

- The LoD of the method is $60 \mu \mathrm{g}$. This method should not be used on gravimetric samples that are expected to experience PM loading less than $60 \mu \mathrm{g}$. Satisfying this LoD may be more challenging in short term samples or low-level ambient samples than in household air pollution or emissions studies. For low PM deposition studies, this technique is not recommended.

- All filters $(\mathrm{N}=12, \sim 5 \%)$ demonstrating a percent error outside the LoA $(\sim \pm 30 \%)$ have a MD of $<280 \mu \mathrm{g}$, which may suggest that confidence in this method will be greatest when mass deposition is expected to be greater than or equal to $280 \mu \mathrm{g}$.

- It is up to the user of this method to decide if the LoA for percent error $(-3.5 \% \pm 29 \%)$ and absolute error $(-5.9 \pm 56 \mu \mathrm{g})$ is acceptable for the user's application.

- While batch number may affect the extraction performance of filters, this is expected to be accounted for by the time-period specific field-blank adjustment. Tracking batch number for the field-blank adjustment analysis may be useful.

- PM composition varies depending on the source. The extraction efficiency determined herein for PM from wood-burning stoves in Lao PDR may differ from other PM sources and make-ups. Other solvents may need to be employed if PM is not soluble in methanol, however, exposure levels during sonication can be high so be careful to use low toxicity solvents and work in 
ventilated work spaces. We suggest evaluating extraction effectiveness of PM from other combustion sources before using this method for filter analysis.

\section{Acknowledgements}

We wish to thank Kirk Smith and L. Drew Hill from the University of California, Berkeley (UCB) for sharing filters for use in the PM extraction tests and final experiment. The filters used in this analysis were collected by Berkeley Air Monitoring Group and UCB under the World Bank-funded cookstove intervention project, which took place in Lao PDR (Contract \#7171980). We would also like to extend our thanks to Katharine Hammond at UCB for advising on the extraction process and results and Ajay Pillarisetti, Drew Hill, and Madeleine Rossanese for input on the manuscript.

\section{Conflicts of Interest}

The authors declare no conflicts of interest regarding the publication of this paper.

\section{References}

[1] Balakrishnan, K., Sambandam, S., Ghosh, S., Mukhopadhyay, K., Vaswani, M., Arora, N.K., et al. (2015) Household Air Pollution Exposures of Pregnant Women Receiving Advanced Combustion Cookstoves in India: Implications for Intervention. Annals of Global Health, 81, 375-385.

[2] Health and Safety Executive (HSE) (1997) General Methods for Sampling and Gravimetric Analysis of Respirable, Thoracic and Inhalable Aerosols. In: Methods for the Determination of Hazardous Substances (MDHS) Guidance, Health and Safety Executive (HSE).

[3] Rosa, G., Majorin, F., Boisson, S., Barstow, C., Johnson, M., Kirby, M., et al. (2014) Assessing the Impact of Water Filters and Improved Cook Stoves on Drinking Water Quality and Household Air Pollution: A Randomised Controlled Trial in Rwanda. PLoS ONE, 9, e91011. https://doi.org/10.1371/journal.pone.0091011

[4] RTI International. (2008) Standard Operating Procedure for Particulate Matter (PM) Gravimetric Analysis. Environmental and Industrial Sciences Division.

[5] USEPA (2017) Method 5: Determination of Particulate Matter Emissions from Stationary Sources. United States Environmental Protection Agency. https://www.epa.gov/sites/production/files/2017-08/documents/method_5.pdf

[6] Drew Hill, L., Pillarisetti, A., Smith, K.R., Delapena, S., Delapena, S., Garland, C., et al. (2015) Air Pollution and Impact Analysis of a Pilot Stove Intervention. Household Energy, Climate, and Health Research Group School of Public Health University of California, Berkeley.

http://ehsdiv.sph.berkeley.edu/krsmith/publications/2015/Lao_MoH_Main_report. pdf

[7] Williams, R., Pasley, T., Warren, S., Zweidinger, R., Watts, R., Stead, A. and Claxton, L. (1988) Selection of a Suitable Extraction Method for Mutagenic Activity from Woodsmoke-Impacted Air Particles. International Journal of Environmental Analytical Chemistry, 34, 137-154. https://doi.org/10.1080/03067318808027411

[8] Deming, W.E. (1943) Statistical Adjustment of Data. Wiley, NY (Dover Publications Edition, 1985). 
[9] Manuilova, E., Schuetzenmeister A. and Model, F. (2014) Mcr: Method Comparison Regression. R Package Version 1.2.1. https://CRAN.R-project.org/package $=\mathrm{mcr}$

[10] Altman, D.G. and Bland, J.M. (1983) Measurement in Medicine: The Analysis of Method Comparison Studies. Journal of the Royal Statistical Society: Series D (The Statistician), 32, 307-317. https://doi.org/10.2307/2987937 